Vol. 30, Issue 4, April 2007

Editorial

167 Size Matters. What Can We Learn from a Small

Randomized Trial?

Saltz, L. (New York, NY)

Original Articles

169 A Randomised Phase II Study of Irinotecan in Combination with 5-FU/FA Compared with Irinotecan Alone as Second-Line Treatment of Patients with Metastatic Colorectal Carcinoma Graeven, U. (Mönchengladbach); Arnold, D. (Halle/Saale); Reinacher-Schick, A. (Bochum); Heuer, T. (Mönchengladbach); Nusch, A. (Velbert); Porschen, R. (Bremen); Schmiegel, W. (Bochum)

177 Effects of a Step-by-Step Inpatient Rehabilitation Programme on Quality of Life in Breast Cancer Patients. A Prospective Randomised Study

Hartmann, U. (Bad Kissingen); Muche, R. (Ulm); Reuss-Borst, M. (Bad Kissingen)

185 Safety and Efficacy of Itraconazole Compared to Amphotericin B as Empirical Antifungal Therapy for Neutropenic Fever in Patients with Haematological Malignancy

Schuler, U. (Dresden); Bammer, S. (Neuss); Aulitzky, W.E. (Stuttgart); Binder, C. (Göttingen); Böhme, A. (Frankfurt/M.); Egerer, G. (Heidelberg); Sandherr, M. (München); Schwerdtfeger, R. (Wiesbaden); Silling, G. (Münster); Wandt, H. (Nürnberg); Glasmacher, A. (Bonn); Ehninger, G. (Dresden)

Clinical Cases

193 Complete Remission and Early Relapse of Refractory Plasma Cell Leukemia after Bortezomib Induction and Consolidation by HLA-Mismatched Unrelated Allogeneic Stem Cell Transplantation Krüger, W.H.; Kiefer, T.; Schüler, F.; Lotze, C.; Busemann, C.; Dölken, G. (Greifswald)

201 Recurrent Low- to Intermediate-Grade Chondrosarcoma of the Thumb with Lung Metastases: An Objective Response to Trofosfamide Debruyne, P. (Leuven/Glasgow); Dumez, H.; Demey, W. (Leuven); Gillis, L. (Rumst); Sciot, R.; Schöffski, P. (Leuven)

206 Asymptomatic Primary Malignant Melanoma of the Esophagus

Gerger, A.; Smolle-Jüttner, F.M.; Samonigg, H.; Wehrschütz, M.; Smolle, J. (Graz)

\begin{tabular}{ll}
\hline KARGER & ( 2007 S. Karger GmbH, Freiburg \\
Fax +4976145207 14 & Artikel (Volltext) und Inhaltsverzeichnisse \\
$\begin{array}{l}\text { E-mail Information@Karger.de } \\
\text { www.karger.com }\end{array}$ & $\begin{array}{l}\text { sowie das vorläufige Inhaltsverzeichnis des nächsten Heftes: } \\
\text { www.karger.com/onk_bk.htm }\end{array}$
\end{tabular}

Band 30, Heft 4, April 2007

Editorial

167 Die Größe ist wichtig. Was können wir von einer kleinen randomisierten Studie lernen? Saltz, L. (New York, NY)

Originalarbeiten

169 Eine randomisierte Studie zu Irinotecan versus Irinotecan in Kombination mit 5-FU/FA in der Zweitlinientherapie von Patienten mit metastasiertem kolorektalen Karzinom Graeven, U. (Mönchengladbach); Arnold, D. (Halle/Saale); Reinacher-Schick, A. (Bochum); Heuer, T. (Mönchengladbach); Nusch, A. (Velbert); Porschen, R. (Bremen); Schmiegel, W. (Bochum)

177 Effekte eines Etappen-Rehabilitationsverfahrens auf die Lebensqualität bei Brustkrebspatientinnen. Eine prospektive randomisierte Studie Hartmann, U. (Bad Kissingen); Muche, R. (Ulm); Reuss-Borst, M. (Bad Kissingen)

185 Sicherheit und Wirksamkeit von Itraconazol im Vergleich zu Amphotericin B als empirische antimykotische Therapie zur Behandlung von neutropenischem Fieber bei Patienten mit hämatologischen malignen Tumoren Schuler, U. (Dresden); Bammer, S. (Neuss); Aulitzky, W.E. (Stuttgart); Binder, C. (Göttingen); Böhme, A. (Frankfurt/M.); Egerer, G. (Heidelberg); Sandherr, M. (München); Schwerdtfeger, R. (Wiesbaden); Silling, G. (Münster); Wandt, H. (Nürnberg); Glasmacher, A. (Bonn); Ehninger, G. (Dresden)

Kasuistiken

193 Vollremission und Frührezidiv einer refraktären Plasmazellleukämie nach Bortezomib-Induktion und konsolidierender, allogener, unverwandter HLA-mismatched Stammzelltransplantation Krüger, W.H.; Kiefer, T.; Schüler, F.; Lotze, C.; Busemann, C.; Dölken, G. (Greifswald)

201 Rezidivierendes niedrig- bis mittelgradig malignes Chondrosarkom des Daumens mit Lungenmetastasen: Objektives Ansprechen unter Trofosfamid Debruyne, P. (Leuven/Glasgow); Dumez, H.; Demey, W. (Leuven); Gillis, L. (Rumst); Sciot, R.; Schöffski, P. (Leuven)

206 Asymptomatisches primäres malignes Melanom des Ösophagus

Gerger, A.; Smolle-Jüttner, F.M.; Samonigg, H.; Wehrschütz, M.; Smolle, J. (Graz) 


\section{Vol. 30, Issue 4, April 2007}

Review Article

209 An Ambiguous Phenomenon of Radiation and Drugs: Recall Reactions

Caloglu, M.; Yurut-Caloglu, V.; Cosar-Alas, R.; Saynak, M.; Karagol, H.; Uzal, C. (Edirne)

Reports of Oncological Societies

\section{Deutsche Fatigue Gesellschaft}

216 PharmaNews / PharmaTicker

219 Meetings and Conferences

168 Imprint

Forthcoming papers are listed on page 220.
Band 30, Heft 4, April 2007

Übersichtsarbeit

209 Eine unklare Begleiterscheinung von Strahlentherapie und Medikamenten: Recall-Reaktionen

Caloglu, M.; Yurut-Caloglu, V.; Cosar-Alas, R.; Saynak, M.; Karagol, H.; Uzal, C. (Edirne)

Mitteilungen onkologischer Gesellschaften

\section{Deutsche Fatigue Gesellschaft}

216 PharmaNews / PharmaTicker

219 Tagungen und Kongresse

168 Impressum

Einen Ausblick auf den Inhalt der kommenden Hefte finden Sie auf Seite 220.

\section{KARGER}

Fax +497614520714

E-mail Information@Karger.de

www.karger.com
() 2007 S. Karger GmbH, Freiburg

Artikel (Volltext) und Inhaltsverzeichnisse

sowie das vorläufige Inhaltsverzeichnis des nächsten Heftes: www.karger.com/onk_bk.htm 